\title{
Walter Benjamin in Lateinamerika: Eine widersprüchliche Erfolgsgeschichte
}

\author{
Horst Nitschack*
}

Die Schriften Walter Benjamins gehören sicher zu den europäischen Texten, die in den beiden letzten Jahrzehnten des 20. Jahrhunderts auf die Theoriebildung innerhalb der Geisteswissenschaften in Lateinamerika, besonders was die Kultur- und Literaturwissenschaften betrifft, mit die nachhaltigsten Auswirkungen gehabt haben.

Wenn wir uns an die Benjamin-Diskussion in Deutschland in den 70er und zu Beginn der 80er Jahre erinnern und an die gegensätzlichen Lektüren seiner Schriften, die sich zwischen den Extremen eines linken, materialistischen und marxistischen Intellektuellen ${ }^{1}$, eines eher an Romantik und Hermeneutik orientierten Philologen ${ }^{2}$ und eines theologischen und der jüdischen Tradition verpflichteten Denkers bewegten ${ }^{3}$, wenn wir uns an diese sowohl polemische wie auch mit großer akademischer Resonanz geführte Debatte erinnern, dürfen wir uns nicht wundern, wenn wir auch in einem intellektuellen und kulturellen sehr anderem Kontext wie dem Lateinamerikas eine Vielzahl von unterschiedlichen Lesarten antreffen, die jeder Möglichkeit einer Systematisierung innerhalb eines Beitrages von dem hier möglichen Umfang widerstehen ${ }^{4}$.

Im Rahmen der hier vorgestellten Untersuchung wird es deshalb nur möglich sein, auf der einen Seite die intensive und umfangreiche Rezeption Benjamins in den unterschiedlichsten intellektuellen, kulturellen und wissenschaftlichen Bereichen, sowohl innerhalb wie auch ausserhalb der Universitäten exemplarisch zu belegen, und auf der anderen Seite einige Hypothesen dazu zu formulieren, wieso dieses nachhaltige Interesse an einem Autor, der $\mathrm{zu}$ seinen Lebzeiten weitgehend ein Unbekannter geblieben war, gerade auch in Lateinamerika geweckt wurde und von so eindrucksvoller Ausdauer ist.

Bekanntlich setzte selbst in Deutschland die Benjamin-Rezeption mit beträchtlicher Verzögerung ein. Die Frankfurter Schule und die Kritische Theorie, deren bedeutendster Vertreter heutzutage in Lateinamerka W. Benjamin ist ${ }^{5}$, wurde nach der Rückkehr nach Deutschland nach dem zweiten Weltkrieg vor allem mit den Namen

\footnotetext{
${ }^{*}$ Universidad de Chile

${ }^{1}$ Wie z.B. bei Jürgen Habermas, Rolf Tiedemann oder Peter Bulthaupt.

${ }^{2}$ Gerhard Kaiser oder xx Menninghaus.

${ }^{3}$ Vor allem Gershom Scholem, aber ein eher theologischer Benjamin auch bei Hermann Schweppenhäuser.

${ }^{4}$ Bei N. Casullo heisst es zur Vielfältigkeit dieser Rezeption: „Por caminos provenientes de la literatura y la estética, de la crítica teórica, de lo filosófico-político, del estudio de lo moderno metropolitano, la obra de Walter Benjamin se fue incorporando al debate de las ideas en América Latina, signado en la última década y media por el extravío de muchos léxicos ordenadores de la realidad."(Casullo: 1993, 13)

${ }^{5}$ Vgl. hierzu das Plakat zu einem Symposium über die Kritische Theorie in México D.F. (Datum) auf dem ein Guerrillero der xxx Bewegung und W. Benjamin im Dialog abgebildet sind.
} 
Max Horkheimer und Theodor Wiesengrund Adorno verbunden und W. Benjamin wurde zu Beginn eher als Randfigur bewertet. Seine nachhaltige Entdeckung als eigenständiger Intellektueller, Theoretiker und Philosoph findet erst gegen Ende der 60 er Jahre statt und war in Deutschland und in Lateinamerika nahezu gleichzeitig ${ }^{6}$.

Eine erste Ausgabe mit acht Aufsätzen W. Benjamins (darunter Über einige Motive bei Baudelaire und Die Aufgabe des Übersetzers) in einer Übersetzung von Héctor A. Murena, wird bereits 1967 in Buenos Aires (Editorial Sur) in der Reihe Estudios alemanes verlegt. Im gleichen Jahr erscheint in Venezuela bei Monte Ávila Sobre el programa de la filosofia futura y otros ensayos (Über das Programm einer zukünftigen Philosophie und andere Essays), wo einige Aufsätze aus Illuminationen versammelt sind. ${ }^{7}$ Von entscheidender Bedeutung jedoch für die Verbreitung des Benjaminschen Denkens in gesamt Lateinamerika waren die vier Bände der Iluminaciones, veröffentlich von Taurus in Madrid, von denen die ersten beiden 1972 erschienen. «Así - stellt Beatriz Sarlo fest -, las traducciones de Taurus marcaron el comienzo del 'fenómeno Benjamin' en la Argentina." Und: Benjamin “se convirtirá [... ] en una moda apasionante." (Sarlo (2000), 42). In Brasilien erscheinen die ersten Benjamin-Uebersetzungen in den gleichen Jahren: Das Kunstwerk im Zeitalter seiner technischen Reproduzierbarkeit wird 1968 in zwei Folgenummern der Revista Cilvilizaçao Brasileira (Nr. 19 und 20, Mai und August 1968) veroeffentlicht ${ }^{8}$.

Allerdings kennt die Benjamin-Rezeption auch den indirekten Weg. Denn in dem Maße, wie im Laufe der 70er Jahre die Benjamin-Rezeption in den Departments für Komparatistik und Kulturkritik an den Universitäten der Vereinigten Staaten an Bedeutung gewinnt, findet sein Denken auch über diesen Weg und vermittelt über das Englische seinen Weg nach Lateinamerika ${ }^{9}$.

Wenn wir einen Blick auf die ersten Arbeiten, die zu W. Benjamin in den 70er Jahren publiziert wurden werfen, ist offensichtlich, dass das Interesse vor allem dem 'linken', eines in der Tradition eines marxistischen aber nicht-dogmatischen Denkens stehenden Intellektuellen gilt (gegen G. Lukács) ${ }^{10}$. Während in Deutschland Benjamin als theoretische Referenz gegen Ende der 80er Jahre an Bedeutung verliert, wird er dagegen in Lateinamerika, vor allem aber in Brasilien und Argentinie, in den

\footnotetext{
${ }^{6}$ Wie G. Wamba Gaviña in ihrem Beitrag zeigt, existierte allerdings bereits in Argentinien in den 30er Jahren ein aussergewöhnlicher Fall einer Benjamin-Rezeption. (Vgl. Wamba Gaviña, 202). In Brasilien gehören zu den ganz frühen Leandro Konder (1967): Os Marxistas e a Arte sowie José Guilherme Merquior (1969): Arte e Sociedade em Marcuse, Adorno e Benjamin.

${ }^{7}$ Cf. Sarlo, Beatriz (1999): Suplemento cultural de Clarín. Reimpreso en : Sarlo, Beatriz (2000) : Siete ensayos sobre Walter Benjamin. p. 41.

${ }^{8}$ Umfangreiche bibliographische Angaben zu den Übersetzungen ins Spanische und Portugiesische finden sich in: Wersching, Klaus-Gunther (2003): Walter Benjamin. Eine Bibliographie.

9 U.a. Jameson, Frederic (1971): Walter Benjamin, or Nostalgia, In: Frederic Jameson: Marxism and Form: Twentieth-Century Dialectical Theories of Literature. Princeton, Princeton University Press, pp. 60-83.

Sontag, Susan (1978): The Last Intellectual. En Susan Sontag (1980): Under the Sign of Saturn. Nueva York. Farrar, Straus, Giroux. pp 107-134. Lunn, Eugene (1982): Marxism and Modernism. An historical study of Lukács, Brecht, Benjamin and Adorno. Regents of the University of California. Traducción (1986): Marxismo y modernismo. Un estudio histórico de Lukács, Benjamin y Adorno. México. Fondo de Cultura Económica.

Buck-Morss, Susan (1991): The Dialectics of Seeing. Walter Benjamin and the Arcades Project.

Cambridge. The MIT Press.

Eagleton

Paul de Man: Conclusion on W. Benjamin

${ }^{10}$ Beatriz Sarlo sieht an einer Stelle die gesamte Benjamin-Rezeption in diesem Kontext: "Sin embargo, sus ideas estéticas se concentran singularmente en un tema: la producción poética de un contendo de verdad que libera energías revolucionarias." (Sarlo: 2000, 43)
} 
intellektuellen Diskussionen und den universitären Seminaren, wie auch in den Veröffentlichungen im Rahmen der Kulturwissenschaften und der Philosophie zu einem der meistzitierten Theoretiker. Das liegt sicher nicht vorrangig an der Vermittlung durch die englischsprachige Welt und an deren Einfluß auf die lateinamerikanische theoretische Diskussion, wenngleich die Bedeutung dieses indirekten Vermittlungsweges vor allem im Bereich der postkolonialen Studien nicht zu übersehen ist ${ }^{11}$. Dennoch gilt, daß in Lateinamerika, sowohl die spanischsprachigen Länder, wie auch Brasilien auf eine von der angloamerikanischen Diskussion völlig unabhängige Benjamin-Rezeption verweisen können.

Im Rahmen der hier vorgelegten Studie wird es, wie bereits erwähnt, nur möglich sein, eine Reihe von Hypothesen aufzustellen, die aufzudecken versuchen, auf welche Besonderheiten und Leistungen des Benjaminschen Denkens diese breite Rezeption und diese intensive Auseinandersetzung mit seinen Schriften in fast allen intellektuellen Kreisen dieser Region zurückzuführen sind. Die lateinamerikanische Kritik (vor allem in Brasilien und Argentinien) laesst keinen Zweifel an der Allgegenwart Benjamins, und sie weisst vielfach darauf hin, dass er Gefahr läuft zu einer bloßen Mode zu verkommen und ein Gefuehl der Übersättigung hervorzurufen. Darauf spielt sowohl Jeanna Marie Gagnebin an, wenn sie ihr Buch História e Narração em Walter Benjamin mit dem Ausruf "Mais um livro sobre Walter Benjamin!” (Gagnebin, 1) beginnen läßt, wie auch der ironische Titel eines Aufsatzes von Beatriz Sarlo: « Olvidar a Benjamin » aus dem Jahr 1995.:

Diese breite und umfassende Rezeption in Lateinamerika im Laufe der letzten 30 Jahre bewegt sich zwischen den Extremen klassischer Benjamin-Exegese und produktiver aber auch eigenwilliger Aneignung, mit der erklärten Absicht einer Anpassung und Aktualisierung des Benjamin'schen Denkens für Lateinamerika ${ }^{12}$, bis zu einem 'recycling', in dem Benjaminsche Versatzstücke relativ willkürlich mit autoritätsfordernder Geste in Argumentationen auftauchen, die sich von seinem Denken bereits vollständig selbstständig gemacht haben. Im negativen Fall kommt es dann zu Erscheinungen, wie sie von Beatriz Sarlo beschrieben werden:

La lectura de Benjamin (y, junto con él como si se
tratara más o menos de los mismo, de Schorske,
Berman, Sennett, De Certeau, Augé, Baudrillard,
entre muchos) ha producido una especie de erosión
teórica que carcome la originalidad benjaminiana
hasta los límites de la completa banalización. Decir
que estamos frente a un caso de empobrecimiento
semántico es poco. Benjamin está ensopado en un
jarabe puramente léxico: se lo cita como si la cita
asegurara, como a veces le aseguraba a Benjamin
después de mucho trabajo compositivo e histórico, la

\footnotetext{
${ }^{11}$ Hier ist vor allem zu denken an Homi K. Bhabhas, The Locations of Culture (1994) und Rey Chows Writing Diaspora: Tactics of Intervention in Contemporary Cultural Studies (1993). Siehe hierzu vor allem: Rolf J. Goebel (1999). "Postkoloniale Kritik und kulturelle Authentizität. Zur Rezeption Walter Benjamins in der amerikanischen Kulturtheorie.“ En: Weimarer Beiträge. 1999, 45 (4) pp. 532-546. Wobei dort unter 'amerikanischer Kulturtheorie' natürlich nur us-amerikanische Theorie verstanden wird. ${ }^{12}$ Martín-Barbero spricht in diesem Zusammenhang von den Chancen der

'Respektlosigkeit'(irreverrencia) dem europäischen Denken gegenüber: „Es una verdadera constumbre la irreverencia con que se suelen leer autores europeos que forman parte del canon. Esa irreverencia nos ha servido para cruzar lecturas de autores y libros cuyo acercamiento era una blasfemia o un sinsentido. Nos posiblitó leer no ordenadamente, minar ciertas cronologías que impiden leer en los destiempos el jeugo de nuevos sentidos y contrasentidos." (Martín-Barbero / Herlinghaus, 165)
} 
producción de un sentido nuevo sobre escenarios diferentes. (Sarlo 2000, 79-80).

Wodurch ist diese in der Tendenz schon inflationäre Aktualität W. Benjamins unter den Intellektuellen Lateinamerikas provoziert worden? In welchem Maße sind die Benjaminschen Text selbst dafür verantwortlich? In welchem Maße waren es die Bedingungen der theoretischen Diskussion vor Ort, die Benjamin zu einer Referenz für ihre Fragestellungen und ihre theoretische Orientierung gemacht haben? ${ }^{13}$

Zweifellos kann der brasilianischen Literaturkritikerin und Übersetzerin Kampff Lage in ihrem Urteil über W. Benjamin beigestimmt werden: «Autor prolífico e de interesses múltiplos, Walter Benjamin produziu uma obra cuja diversidade talvez constitua a sua marca mais evidente.” (Kampff Lage, 21) Die Diversität des Benjaminschen Ouevres ist sicher eine der Voraussetzungen für seine umfassende und vielfältige Rezeption. Allerdings bleibt auch dieses Urteil nicht unwidersprochen, wenn der - ebefalls brasilianische - Literaturwissenschaftler Márcio Seligmann-Silva in Ler o Livro do Mundo: Walter Benjamin: Romantismo e Crítica Poética feststellt, dass eine "unidade da obra de Benjamin" besteht, die "assenta-se na sua concepção romântica da linguagem como a unidade entre o âmbito mágico e o profano." (Seligmann-Silva, 219).

Vor dieser Alternative, sich zwischen einem Benjamin höchster Diversität und dem eines einheitlichen Denkens entscheiden zu müssen, schlage ich hier vor, sieben unterschiedliche Linien oder Profile in seinem Denken zu berücksichtigen, mit Hilfe derer unterschiedliche Motivationen und Begründung für seine Rezeption in Lateinamerika herausgearbeitet werden können, Motive und Gründe, die sich nicht gegenseitig ausschliessen, sondern die sich auf unterschiedliche Weise, je nach dem Interesse und der theoretischen Position der Leser, ergänzen können.

1. Das Interesse an einem nicht-dogmatischen marxistische Denken und die Distanzierung von einem auf die kommunistische Partei zentrierten Marxismus. Damit setzte vielfach die erste 'Entdeckung' W. Benjamins ein (Konder, MartínBarbero).

2. Benjamins Beitrag zu einer Theorie der Moderne (R. Ortiz)

3. Benjamin als Theoretiker der Großstadt (Beatriz Sarlo, W. Bolle)

\footnotetext{
${ }^{13}$ Leider ist es in diesem Rahmen auch nicht möglich eine nach Ländern differenzierte BenjaminRezeption vorzustellen. Brasilien und Argentinien nehmen jedoch allem Anschein nach hierbei ein führende Rolle ein. Zur Rezeption in Brasilien liegen auch bereits verschiedene Arbeiten vor. Besonders die Untersuchung von Gunter Pressler (1995): Benjamin, Brasil: Die Walter Benjamin - Rezeption in Brasilien. Tese de doctorado, São Paulo (USP) sowie der Aufsatz des gleichen Autors: Pressler, Günter $\operatorname{Karl}(1993)$ : „El perfil de los hechos. Sobre la recepción de Walter Benjamín en el Brasil”. En: Fehrmann, Silvia y Gabriela Massuh (1993): Sobre Walter Benjamín. Vanguardias, historia, estética y literatura. Una visión latinoamericana. Buenos Aires. Alianza Editorial /Goethe-Institut Buenos Aires. pp 223-233. Außderdem die Studie von Rouanet, Sérgio Paulo (1997): „Die Rezeption der deutschen Philosophie in Brasilien: der Fall Walter Benjamin“. In: Andreas Boeckh; Rafael Sevilla (Hrsg.): Bestandsaufnahme und Perspektiven der deutsch-brasilianischen Beziehungen : 1. Fachtagung der ADLAF-Arbeitsgruppe Brasilien (Konstanz, 21.-23. Juni 1996). Frankfurt am Main. pp. 31-61. In anderen Ländern, wie z. B. in Peru ist hingegen diese Rezeption viel zurückhaltender.. So fand in Lima an der Universidad San Marco vom 3. bis zum 5. November 2004 das Seminar La filosofía del fragmento statt, das im Internet als ,el primer seminario dedicado al pensador berlinés en el Perú” angekündigt wurde. (http://www.ccsm-unmsm.edu.pe/boletin/055.htm vom 20.1.2005)
} 
4. Benjamins Beitrag zu einer Literaturkritik und zur Medientheorie (MartínBarbero)

5. W. Benjamin als Theoretiker einer Wiederentdeckung der Geschichte (P. Oyarzún) wie auch als Referenz für die die Memoria-Diskussion und Testimonial-Texte (Nelly Richard)

6. Benjamins Beitrag zur Erneuerung und Neuformulierung eines hermeneutischen Denkens; Rezeptionstheorie, Theorie der Übersetzung und Interkulturalität (P. Oyarzún, M. Seligmann-Silva, Kampff-Lage)

7. Benjamins Beitrag zur Kulturkritik und postkolonialen Kritik (Beatriz Sarlo, Martín-

Barbero)

Zweifellos koinzidieren die 'Angebote' des Benjaminschen Denkens mit unterschiedlichen politischen und kulturellen Konjekturen in den lateinamerikanischen Ländern, die ihrerseits die Dispositionen für die jeweiligen Rezeptionen geschaffen haben. An erster Stelle ist dabei sicher der Benjaminsche Entwurf eines integrativen Denkens, der die festen Grenzen zwischen den Disziplinen der Humanwissenschaften, besonders Literaturkritik, Philosophie, Soziologie aufzuheben versucht, zu erwähnen. Dieses Oszillieren zwischen Philosophie und Literaturkritik, zwischen politischem und theologischen Denken, zwischen Hermeneutik und Dekonstruktion avant la lettre kommt einer Tradition innerhalb der Humanwissenschaften entgegen, die für Lateinamerika charakteristisch ist und die nicht das hohe Maß der Ausdifferenzierung europäischer Geisteswissenschaften kennt. Die Tradition des Essay, eines offenen Diskurses, der soziologisch, politisch, literarisch, anthropologisch, oder wie auch immer, je nach Bedarf argumentieren kann (heute wird diese Form des Schreibens vorzugsweise mit dem Attribut 'hybrid' versehen), ist in Lateinamerika seit dem 19. Jahrhundert ausgeprägt. In seiner Verweigerung, die konventionellen Ausgrenzungen zwischen den Diskursen zu wiederholen, kommt das Benjamin' sche Denken dieser Tradition fraglos entgegen. Inter- und Transdiziplinaritaet, die heute allerorts entdeckt wird, ist den Benjamin'schen Texten von Beginn an eigen, wie auch ihr Anspruch, nicht nur in einem hochspezialiserten akademischen Raum zu zirkulieren, sondern in einer breiteren intellektuellen Oeffentlichkeit Aufnahme zu finden. Die Unabgeschlossenheit und Offenheit seiner Positionen, die sich am eindeutigsten im fragementarischen Charakter seines Schreibens, in der Vielfalt seiner Zitate und in der Reichhaltigkeit der Intertexte manifestiert, trifft auf eine weitere intellektuelle Tradition Lateinamerikas, die zuweilen als 'eklektisch' (Leopoldo Zea) und unsystematisch oder heutzutage - eine provozierende Idee der brasilianischen Modernisten der 20er Jahre aufgreifend - als 'antropophag' benannt wird.

Auch das Benjaminsche Verfahren Gedankensplitter und Fragmente aus anderen Texten für seine eigenen Argumentationen zu nutzen und sie aus der Autorität des ihnen eigenen Systems freizusetzen korrespondiert mit einer lateinamerikansichen Schreibweise, die bereits mit Sarmiento einsetzt und sich ueber Rodó bis in die Gegenwart fortsetzt. ${ }^{14}$

Neben diesen allgemeinen intellektuellen und kulturellen Voraussetzungen, die zumindest einen Hinweis auf die Gründe für die Bereitschaft der Rezeption der

\footnotetext{
${ }^{14}$ Gleiches gilt für die intensive Nietzsche-Rezeption während der ersten Jahrzehnte des 20. Jahrhunderts: der fragementarische Chrakter seines Philosophierens erlaubte die unterschiedlichsten Anschlussmöglichkeiten für ein lateinamerikanisches Denken, .
} 
Benjamin'schen Schriften geben, die nun bereits über Jahrzehnte anhält, treten noch verstärkend die besonderen historischen und politischen Umstände der späten 60er und der 70er Jahre. In gesamt Lateinamerika koinzidierte diese Rezeption, zumindest innerhalb der intellektuellen Linken, die als erste auf Benjamin aufmerksam wurde, mit einer Distanzierung vom kubanischen Modell des Sozialismus (implizit damit auch mit einer weiteren Distanzierung von der Sowjet Union) und damit von einem dogmatischen partei-zentrierten Marxismus. In Brasilien, Chile und Argentinien fällt die erste Epoche dieser Rezeption außerdem mit den repressiven Phasen der Militärdiktaturen zusammen Hieraus ergeben sich mindestens zwei sich komplementierende zusätzliche Gründe für eine Benjamin-Rezeption. Sie liegen in der Notwendigkeit eines Überdenkens 'klassischer' marxistischer Positionen, da sie

1. zu einer politischen Wirklichkeit geführt haben, die weder in der SowjetUnion noch in Kuba ${ }^{15}$ weiterhin mit dem Anspruch auf Modell-Charakter auftreten können, bzw.

2. in keinem der drei genannten Länder in der Lage waren, die Militärdiktaturen zu verhindern, bzw. einen breiten, von großen Anteilen der Bevölkerung ('Massen') getragenen Widerstand zu organisieren. hinzu:

Dem fügt sich noch ein dritter, vielleicht überhaupt der ausschlaggebende Grund

3. die Kritik des Modernisierungsprozesses, sowohl nach sowjet-russischem, wie auch nach okzidental - us-amerikanischem Modell. Für einen bedeutenden Teil lateinamerikanischer Intellektueller erscheint keines der beiden Modelle in den 70er Jahren einen gangbaren Weg für diese Region zu bieten (Abkopplungstheorien). Dem sowjetischen Modell fehlt die breite Unterstützung durch die Bevölkerung und das usamerikanische Modell, das sich im Laufe der letzten Jahrzehnte des 20. Jahrhunderts durchsetzen wird, ist auf die Unterstützung durch die Politik der Militärdiktaturen angewiesen oder wird zumindest mit dieser Politik identifiziert. Denn es sind diese Militärdiktaturen, die die wirtschaftliche, z.T. auch administrative Modernisierung (bei konsequentem Ausschluss der politischen Modernisierung, sprich Demokratisierung) forcieren.

Hier bietet Benjamins Kritik des (sozialdemokratischen implizit auch dogmatisch-marxistischen) Fortschrittsdenkens, das gleichwohl nicht auf Modernisierung verzichtet, sondern, wie besonders in seinem Kunstwerk-Aufsatz, die faschismuskritische Potentialität der Technik aufdeckt, vielversprechende Ansatzpunkte, um eine andere Modernität zu denken.

Die Suche nach einer anderen Modernität hält auch an, wenn die Militädiktaturen im Prozess der Öffnung (abertura: Brasilien) und des Übergangs (transición: Argentinien, Chile) in Demokratien überführt werden: Das sich durchsetzende neoliberale Modell hat eine erneute soziale und kulturelle Ausschließung von Subalternen zur Konsequenz und droht eine Geschichtsschreibung zu favorisieren, die sich mit den Siegern identifiziert und in denen die Besiegten und die Opfer dem Vergessen anheimfallen. Hier bietet sich nun der Rekurs auf das Benjamin'sche Geschichtsmodell an, das als Aufforderung gelesen wird und als eine Mahnung, die Erinnerung an die vergessene und unterdrückte Vergangenheit wach zu halten.

Als letzter dieser allgemeinen strukturellen Gründe für die Rezeption W. Benjamins ist an die vehemente Urbanisierung und die Herausbildung einer durch die Medientechniken beschleunigten Kulturindustrie $\mathrm{zu}$ denken, beides Folgen des

\footnotetext{
${ }^{15}$ Vor allem der berühmte 'Fall Padilla' 1971, der zu einer politischen Distanzierung eines großen Teiles der lateinamerikanischen Linken vom kubanischen Sozialismus führte.
} 
Modernisierungsprozesses, die zu den wohl einschneidendsten im Alltag erfahrbaren Veränderungen geführt haben. Auch in diesen beiden Fällen bieten die Schriften Benjamins vielversprechende Hinweise, diese gesellschaftlichen und kulturellen neuen Wirklichkeiten zu denken.

Im Folgenden wird anhand der oben vorgeschlagenen sieben Punkte ein etwas detaillierteres Panorama der Benjamin-Rezeption vorgestellt werden, das allerdings bei weitem nicht erschöpfenden oder umfassenden Charakter hat und das trotz seiner Bemühungen repräsentativ zu sein, dennoch von einer gewissen Zufälligkeit nicht frei ist..

\section{Benjamin als Vertreter einer nicht dogmatischen marxistischen Theorie:}

Für diese Rezeption, die, wie bereits gesagt, Ende der 60er Jahre einsetzt, ist Benjamin in der Regel neben Adorno ein Vertreter der Frankfurter Schule. MartínBarberos erste Begegnung mit Benjamin kann hier sicher als beispielhaft zitiert werden. "Trabajaba yo entonces (otoño del año $69 \mathrm{HN}$ ) sobre las transformaciones del marxismo que venían tanto del 'oriente' como del 'occidente' - Adam Schaff, Karel Kosic, Lous Althusser, Lucien Goldman - y en el texto de Benjamin descurbrí otro marxismo, el más otro de todos." (Martín-Barbero, 11). Ähnliches gilt für die Entdeckung der Kritischen Theorie in Brasilien Ende der 60er Jahre von Autoren wie Leandro Konder und José Guilhermo Merquior, wobei in diesen Fällen W. Benjamin allerdings noch nicht die Sonderstellung innerhalb der Frankfurter Schule zukommt, wie es dann später der Fall sein wird .

\section{W. Benjamin als Repraesentant einer Theorie der Moderne:}

Im Verlauf des letzten Drittels des 20. Jahrhunderts werden die meisten lateinamerikanischen Staaten immer stärker in den internationalen Modernisierungsprozess einbezogen, der dann ab den 80er Jahren als 'Globalisierung' diskutiert wird. Renato Ortiz ist wahrscheinlich der brasilianische Soziologe bei dem in A moderna tradiçao brasileira die Verweise auf Benjamin und dessen Reflexionen zur Moderne am häufigsten sind. Erstaunlichweise gilt dies jedoch nicht für eines seiner folgenden Bücher, in dem der Modernisierungsprozess in Frankreich im 19. Jahrhundert untersucht wird und wo am ehesten ein Verweis auf Benjamins Paris, Hauptstadt des 19. Jahrhunderts hätte erwartet werden koennen ${ }^{16}$. A moderna tradiçao brasileira (1988) verweist auf die Frankfurter Schule und auf W. Benjamin sowohl im Zusammenhang der These von der Autonomie der Kunst (s. 21) wie auch der Entstehung der Kulturindustrie im 20. Jahrhundert (S. 15). Ein eingehendere Auseinandersetzung mit Benjamin legt Ortiz aber dann erst zwölf Jahre spaeter in Walter Benjamin e Paris. Individualidade e trabalho intelectual vor ${ }^{17}$. Hier wird vorgeführt, wie Benjamin mit Konzepten wie 'Flaneur' oder 'Boheme' den Modernisierungsprozess im Frankreich des 19. Jahrhunderts erfasst, ohne dass allerdings von R. Ortiz dabei ihr Charakter als 'dialektische Bilder' wirklich erkannt wird, so dass man als Leser den Eindruck hat, Ortiz antwortet auf die breite BenjaminRezeption, ergänzt sie durch einige erläuternde Ausführungen, und versucht die Benjamin-Begeisterung vieler Leser etwas zurechtzurücken, indem er ihn in den

\footnotetext{
${ }^{16}$ Ortiz, Renato (1991): Cultura e modernidade. São Paulo. Brasilense.

${ }^{17}$ In Ortiz (2000 b) ist die spanische Version dieses Artikels veröffentlicht.
} 
breiteren Zusammenhang der soziologischen Modernitäts-Diskussion stellt und darauf hinweist, dass bei ihm steht, was auch andernorts zu lesen ist, aber tatsächlich auch nicht mehr: "Sociologicamente, a pergunta que se pode fazer é a seguinte: porque o flaneur suge apenas no século XIX? Quais sao as transformaçoes que permitem o seu advento? Embora Benjamin nao formula explicitamente a questao, a resposta encontrase ao longo de toda sua obra: ele é fruto da modernidade." Eine solche Antwort fällt allerdings für den überzeugten Benjamin-Leser weit hinter die Bedeutung zurück, die der Figur des Flaneur für Benjamin zukommt. Für unsere Argumentation scheint mir dabei besonders wichtig zu sein, dass es - ob zurecht oder zu unrecht - auch ein durchaus sehr 'sachliche' Benjamin-Rezeption gibt, die sich vor einer Überbewertung hüten will und die versucht, ihn an den Ort zu stellen, wo er dem Autor zufolge hingehört.

\section{W. Benjamin, Theoretiker der Grossstadt:}

Hier ist vor allem an die argentinische Literatur- und Kulturkritikerin Beatriz Sarlo zu denken, wenngleich von ihr der Autor der Säedtbilder und von Paris, Hauptstadt des 19. Jahrhunderts durchaus auch als Begründer eines neuen Paradigmas von Kulturkritik begriffen wird, wenn sie von der 'método Benjamin' spricht: "En efecto el 'método Benjamin' (si se permite esta expresión inusual para su objeto) es, como la estrategia surrealista, una aproximación entre dos registros que, cada uno en sí mismo ha perdido su verdad, pero cuya contraposición instituye un sentido." (Sarlo 2000, 27). Dass sie es war, die an Benjamin vor allem die 'urbane Dimension' entdeckt hat, bestätigt auch Martín-Barbero:

Beatriz Sarlo lee a Benjamin en un ejercicio
desmitificador de las generalizaciones deformantes y
hermeneuta de las verdaderas pecularidades de su
pensamiento sobre la ciudad. Pues el París que
interesó a Benjamin no fue el de moda sino 'el
escenario cultural indispensable para entender algo
que no era Paris: la prehistoria del siglo XX en las
formas de la mercancía del XIX', las claves
culturales del movimiento del arte y de las
mercancías en la modernidad." (Martín-Barbero
1999, 20-21). ${ }^{18}$

Gleichzeitig aber auch ist sie es, die vor einer verdummenden Benjamin-Manie warnt, bei der seine Begriff und Denkfiguren für alles herhalten müssen:

Un murmullo donde las palabras flâneur y flânerie se usan como inesperados sinónimos de prácticamente cualquier movimiento que tenga lugar en los espacios públicos. Se habla de la flânerie en ciudades donde, por definición, sería imposible la existencia del flâneur. (Sarlo 2000, 78).

\footnotetext{
${ }^{18}$ Eine derartige Benjamín-Lektuere geht deutlich über diejenige von R. Ortiz hinaus.
} 
An dieser Stelle muss natürlich auch die umfangreiche Untersuchung des deutsch-brasilianischen Literaturwissenschaftlers Willi Bolle Physignomik der modernen Metropole: Geschichtsdarstellung bei Walter Benjamin erwähnt werden, die 1994 in Deutschland und im gleichen Jahr auch auf Portugiesisch in Brasilien erschien. W. Bolle macht in dieser Arbeit explizit den Versuch, mit W. Benjamin von der Peripherie her zu denken, von einer Peripherie aus, die den zerstörenden Kräften der Modernisierung noch weitaus schutzloser ausgeliefert zu sein scheint, als die Zentren, die diese Kräfte in die Welt entlassen haben: “...die Signale des Schiffbruchs, die der Kritiker an der Metropole des 19. und beginnenden 20. Jahrhunderts wahrgenommen hatte, (sind) im Boot der Menschheit stärker geworden (...) vor allem an der Peripherie." (Bolle 1994 b, 34).

So hat sich dann auch seine Studie zur Aufgabe gemacht, im Benjaminschen Werk "ein Potential für die Erkenntnis einer unvollendeten bzw. gescheiterten Moderne" (19) freizulegen. Denn, so W. Bolle: "Bestimmte Beobachtungen und Reflexionen in seinen Schriften warten drauf, von einem anderen Fluchtpunkt her als dem europäischen aus ihrem Zusammenhang herausgesprengt und nach einem neuen Bauplan zusammengefügt zu werden. Die vorliegende Arbeit, entstanden in einer Metropole der Dritten Welt (São Paulo), versteht sich als ein Schritt in diese Richtung." (Bolle 1994 b, 21). Damit wird von W. Bolle ein Programm skizziert und eine Möglichkeit der Benjamin-Rezeption, die für eine Tendenz der lateinamerikanischen Benjamin-Lektüre stehen: ihr Kennzeichen ist eine Verkopplung oder eine Konstellation von Benjamin'scher Reflexion und lateinamerikanischem Diskurs, die, wenn auch manchmal $\mathrm{zu}$ etwas bedenkliches Kurzschlüssen führend (wie oben von Beatriz Sarlo kritisiert), doch in der Regel ein sehr kreatives kritisches Denken in Bewegung gesetzt haben, wie wir es im Fall von Nelly Richard, aber auch von MartínBarbero exemplarisch vorgestellt bekommen.

\section{Literaturkritik und Beitrag zu einer Medientheorie:}

In der brasilianischen Literaturkritik ist Luis Costa Lima sicher der Theoretiker bei dem der Bezug auf die deutschsprachige Philosophie und Philologie am umfangreichsten ist, das gilt sowohl für die Repräsentanten der Frankfurter Schule wie auch für die Rezeptionsästhetik von Wolfgang Iser und Jauss. In diesem Zusammenhang ist auch W. Benjamin eine immer wieder zitierte Referenz, was aber nicht bedeutet, dass er je eine besondere Stellung in L. Costa Limas literaturwissenschaftlichen Untersuchungen eingenommen hätte, sondern er ist einer unter den vielen Namen in den an Theorieverweisen reichen Veröffentlichungen dieses brasilianischen Literaturwissenschaftlers.

Eine besondere Rolle hingegen nimmt W. Benjamin nach dessen eigenen Bekundungen in der theoretischen Entwicklung Jesús Martín-Barberos ein. Für ihn gilt: Benjamin "va a ser el primero en elaborar una concepción no conservadora de la masa.", gegen Tocqueville, Tarde und Le Bon, (Martín-Barbero / Herlinghaus, 16) Sein Buch Medios y Mediaciones, ist, wie er selbst schreibt, massgeblich unter der Einwirkungen seiner zweiten Benjamin-Lektüre geschrieben (Martín-Barbero / Herlinghaus, 18). Stand seine erste Benjamin-Lektüre ("Der Autor als Produzent") im Zeichen des Pariser Mai 1968 (Martín-Barbero / Herlinghaus, 11), so war die zweite, Anfang der 80er Jahre, im nachfranquistischen Spanien durch die vier Schlüsseltexte: "Das Kunstwerk im Zeitalter seiner technischen Reproduzierbarkeit", "Eine kleine Geschichte der Phptographie", "Erfahrung und Armut" und "Thesen zum Begriff der 
Geschichte" bestimmt. (Martín-Barbero / Herlinghaus, 14-15). Vor dem Hintergrund der Lektüre dieser Benjaminschen Schriften reformuliert Martín-Barbero das Verhältnis von popularem Gedächtnis (memoria popular)und dem Imaginären der Massen (imaginario de masas) (S. 16) und geht den Voraussetzungen der Massenkultur in der Volkskultur (de lo masivo en lo popular) nach.

Benjamin ist fur ihn der Ausgangspunkt für den Entwurf einer postmodernen Kulturkritik, einer Kritik, dessen ist sich Martín-Barbero bewusst und hierin steht er hier stellvertretend für eine weit verbreitete Rezeptionsweise, die Benjamin in Argumentationszusammenhängen verwendet, die aus seinen eigenen Texten nicht unbedingt hervorgehen:

\begin{abstract}
Es una verdadera costumbre la irreverencia con que se suelen leer autores europeos que forman parte del canon. Esa irreverencia nos ha servido para cruzar lecturas de autores y libros cuyo acercamiento era una blasfemia o un sinsentido. Nos posibilitó leer no ordenadamente, minar ciertas cornológicas deterministas que impiden leer en los destiempos el juego de nuevos sentidos y contrasentidos. (MartínBarbero / Herlinghaus, 165)
\end{abstract}

Benjamin wird bei Martín-Barbero, und nicht nur bei ihm, zu einem postmodernen Denker avant la lettre, ein Denker der der Diaspora angehörte, ein Denker des Nomadischen, der Nicht-Sesshaftigkeit, bei dem die Auflösung des Zentrums zur Methode wurde (Martín-Barbero / Herlinghaus, 15):

Yo encontré una validación de la productividad del
,desorden, latinoamericano precisamente en
Benjamin, ese alemán que pensaba desde los
márgenes, pensaba lo grotesco, la moda, la
fotografía, los espejos, los pasajes, conectando
dimensiones que nadie había conectado. Por eso me
disgusta el Adorno que le exige que haga sistema.
Benjamín es aquel pensador moderno, de izquierda,
que ha sabido indagar las conexiones más secretas y
densas entre lo cultural y lo político, entre lo social y
lo estético. (Martín-Barbero / Herlinghaus, 165-166).

\title{
5. Die Wiederentdeckung der Geschichte, der Erinnerung und einer Gedächtniskultur:
}

Obwohl Historia e Narração em Walter Benjamin von Jeanne Marie Gagnebin im engeren Sinn nicht zu dem hier vorgestellten Textkorpus gehört, da es sich um eine Übersetzung von Histoire et Narration chez Walter Benjamin, Paris 1994 (Harmattan) handelt, und die zahlreichen Übersetzungen wissenschaftlicher Literatur zu Benjamin ins Spanische und Portugiesische und deren Veröffentlichungen in lateinamerikanischen Verlagen hier nicht berücksichtigt worden sind, soll in diesem Fall eine Ausnahme gemacht werden, denn die Autorin gehört aufgrund ihrer akademischen Einbindung eher zur brasilianischen Benjamin-Rezeption als zur französischen oder deutschen (ihre 
Dissertation wurde auf Deutsch veröffentlicht ${ }^{19}$ ). Andererseits weist dieses Beispiel aber auch darauf hin, dass eine regionale oder gar nationale Zuordnung einer BenjaminRezeption zuweilen zufällig oder sogar willkürlich ist.

Auch J.M. Gagnebin Buch ist ein Vorschlag, wenn auch nicht eine Einheit im Benjamin'schen Denken herauszustellen, so doch den Bruch zwischen seiner bereitwilligen Öffnung der Modernen gegenüber auf der einen Seite, sowohl in ihrem Erzählen (Proust und Kafka) wie auch in ihrer Akzeptanz der Technik (die technische Reproduzierbarkeit: Fotografie und Film), und dem nostalgischen ja melancholischen Grundzug, der immer wieder bei ihm aufscheint, auf der anderen Seite, als Resultat einer Spannung zwischen der Idee des ,Ursprungs' und der ,Moderne' zu denken (Gagnebin, 7-8): „Tal recorrência da dinâmica do Ursprung nestes contextos tão diversos deveria permitir situar esse pensamento além das alternativas habituais (Theologie gegen Materialismus, konservatives Denken gegen revolutionäres, Nostalgie gegen Avantgarde $\mathrm{HN}$ ) - e, igualmente, do romantismo revolucionário - e ler a filosofia da história e a filofofia da linguagem de Benjamin como uma reflexão centrada na modernidade, no profundo co-pertencimento do eterno e do efêmero.“ (Gagnebin, 9).

Auch J.M. Gagnebin hat einen, universellen' Benjamin im Blick, sowohl hinsichtlich der Bedeutung seiner Geschichtsphilosophie für die Moderne, wie auch hinsichtlich einer Theorie des Erzählens und dem Paradoxon, dass das was wirklich erzählt werden muß, nicht zu erzählen ist:

\footnotetext{
Se a lembrança se contenta em conservar piamente o passado numa fidelidade inquieta e crispada, ela se torna, sub-repticiamente, infiel a ele porque negligencia o essencial: o que havia nele de renovação e que só pode repetir-se sendo outro, criação e diferença. Essa estrutura paradoxal do lembrar criador e transformador [...] funda a concepção benjaminiana de uma escrita da historia ao mesmo tempo destrutora e salvadora." (Gagnebin, 105) Letzter Bezugspunkt an dem sich für sie diese Benjamin'sche Einsicht bewährt, sind aber nicht (durchaus denkbare) Beispiele aus der lateinamerikanischen Geschichte, sondern die Shoah (Gagnebin, 107). (E)la [a historiografia da Shoah $\mathrm{HN}$ ] se vê confrontada à imperiosa necessidade de lutar contra o esquecimento pelo trabalho de rememoração ou de testemunho e, ao mesmo tempo, à impossibilidade de encontrar as palavras que digam $o$ horror sem nome, em particular à impossibilidade de dar suas razões e de formular explicações adequadas a seu respeito. (107).
}

Aber vielleicht ist dieser Verweis auf die Shoah doch nicht so ,universell' wie er erscheinen mag und birgt - beabsichtigt oder unbeabsichtigt - einen Verweis auf die lateinamerikanische Geschichte der letzten Jahrzehnte, vor allem auf die Jahre der Militärdiktaturen. Der argentinische Literaturwissenschaftler Fernando Reati stellt sich diese Frage hinsichtlich der Erzählbarkeit von Gewaltanwendungen während der Zeit der argentinischen Militärdiktatur und stellt fest, dass anders als in Kolumbien, wo in der Literatura de la violencia "der Versuch gemacht wurde, die Gewalt darzustellen und

\footnotetext{
${ }^{19}$ Gagnebin, Jeanne Marie (1976): Zur Geschichtsphilosphie Walter Benjamins: die unabgeschlossenheit des Sinnes. Erlangen, Palm und Enke.
} 
eine Antwort auf das, Warum' zu geben, in Argentinien, wie angesichts der Shoah, nur noch die blosse Frage, warum' gestellt werden konnte, ohne die Hoffnung auf irgendeine Antwort ${ }^{20}$.

Der Text, der vielleicht die größte Auswirkung in der Benjamin-Rezeption gehabt hat, vor allem seit dem Ende der 80er Jahre, der Text, der wahrscheinlich am häufigsten zitiert ist und von dem verschiedene Gedanken unter den BenjaminVerehrern so etwas wie ,geflügelte Worte' geworden sind, sind die Thesen Über den Begriff der Geschichte oder die Geschichtsphilosophischen Thesen ${ }^{21}$. Mit einem 1995 signiertem Vorwort und einer anspruchsvollen philosophischen Einführung veröffentlicht Pablo Oyarzún Robles eine sorgfältige Übersetzung dieses Textes, der sowohl die unterschiedlichen deutschen Varianten, wie auch die französische Version berücksichtigt ${ }^{22}$. Bemerkenswert an der Einführung sind in unserem Zusammenhang zwei Dinge: 1. Die Selbstverständlichkeit mit der Oyarzún beim lateinamerikanischen Leser die Kenntnis der historischen und politischen Umstände voraussetzt, in deren Zusammenhang diese Thesen zu lesen sind und auf die diese reagieren und 2. die Verweise mit denen diese Thesen in den theoretischen und philosophiegeschichtlichen Kontext, der um die Begriff ,Erfahrung', Geschichte' und Faktizitaet' kreist eingebunden werden, zielen in keinem Moment auf eine Aktualisierung oder auf eine Adaptierung Benjamin'scher Geschichtsphilosophie auf lateinamerikanische Umstände. Diese Aufgabe bleibt dem Leser selbst überlassen. Hierin unterscheidet sich Oyarzún von der chilenischen Literatur- und Kulturkritikerin Nelly Richard oder der Argentinierin Beatriz Sarlo von denen Martín-Barbero sagt: "Nelly Richard me ha ayudado a encontrar en Benjamin el decifrador de los destiempos, y Beatriz Sarlo a leer las contradictorias señales de la presencia de Benjamin a la hora de pensar nuestras experiencias de ciudad." (Martín-Barbero / Herlinghaus, 19). Anders als diese beiden Autorinnen, die ähnlich wie Willi Bolle, eine Relektüre Benjamins von der Peripherie aus vornehmen, wird Benjamin bei Oyarzún in einen universellen philosophischen Diskurs gestellt.

Das erste Kapitel von Nelly Richards La Insubordinación de los Signos ist eine Hommage an Walter Benjamín. Nelly Richards dekonstruktivistischer Umgang mit Texten und auch mit denen Benjamins hätte wahrscheinlich auch diesen überrascht und vielleicht hätte er ähnlich reagiert wie eine andere chilenische Literaturkritikerin, Adriana Valdés, in einem Interview mit N. Richard:

\begin{abstract}
A mí siempre me ha pasado con los textos de Nelly que, por un lado, me interesa mucho estar citada ahí porque ella les da una existencia a las palabras recogidas que las palabras solas, vagabundas, quizás no tenían; pero, por otro lado, me siento siempre un poco tironeada por el texto. Se produce siempre un roce, una pequeña fricción entre lo que está incorporado como cita y el texto mismo." (N. Richard, 99).
\end{abstract}

\footnotetext{
${ }^{20}$ Fernando Reati (1992): Nombrar lo innombrable. Buenos Aires: Editorial Legasa.

${ }^{21}$ Benjamin, Walter (1980): Über den Begriff der Geschichte. In. Benjamin, Walter (1980): Gesammelte Schriften, Werkausgabe, Bd. 2, Frankfurt: Suhrkamp. pp. 691- .

${ }^{22}$ Diese Übersetzung mit ihren Anmerkungen könnte vorbildhaft für zukünftige Übersetzungen sein. Eine kritische Beurteilung und Durchsicht der vorliegenden Benjamin-Übersetzungen, die sich durch Ungenauigkeiten und Uneinheitlichkeit der Begrifflichkeiten auszeichnen, wäe eine wichtige Aufgabe einer Benjamin-Philologie in Lateinamerika.
} 
Diese Reibung und diese Spannung in denen die Zitate im Verhältnis zu dem Kontext stehen, aus dem sie genommen sind, ist durchaus intentional und Teil der Appropriation oder der, antropophagischen' Vereinnahmung, die N. Richard mit den von ihr zitierten Texten vornimmt. Hier kann sie sich durchaus auch auf Benjamin berufen und seine Politik, die Potentialität von Textfragmenten durch die Gegenwart und ihre Argumentationszusammenhänge bestimmen $\mathrm{zu}$ lassen und nicht durch eine zweifelhafte historische Authentizität, die sich zu einer undurchschaubaren Autorität aufschwingt. Ein Beispiel für diese Strategie der De- und Rekontextualisierung Benjamin'schen Denkens ist, wenn sie dessen Reflexion zur Ästhetik der Fotografie wörtlich auf Chile überträgt. Anhand von Atgets Aufnahmen, die Benjamin mit denen eines Tatorts verglich stellt im weiteren die Frage: "Aber ist nicht jeder Fleck unserer Städte ein Tatort? Nicht jeder ihrer Passanten ein Taeter? Hat nicht der Photograph Nachfahr der Augurn und der Haruspexe - die Schuld auf seinen Bildern aufzudecken und den Schuldigen zu bezeichnen?" (Benjamin, 385). Diese Benjamin'schen Reflexionen überträgt N. Richard unmittelbar auf die Bilder des chilenischen Fotografen E. Dittborn, mit denen er auf die Gräueltaten der Militaerdiktatur reagierte:

\begin{abstract}
La obra de Dittborn armaba la semejanza entre los retratos abandonados en la vitrina fotográfica y los cuerpos tirados por la máquina de desidentidad (tortura + anonimato) en los cementerios clandestinos. Estas fueron dos de las situaciones chilenas en las que el retrato pasó a ser la imagen de una delación fotográfica: la de la foto como 'lugar del crímen' en la que, según Benjamín, debe el artista 'descubrir la culpa...y señalar el culpable' (...), rastreando las huellas técnicas de la maquinación visual orquestada por el aparato serializador que dicta la sentencia colectiva." (N. Richard, 21).
\end{abstract}

In der Verkoppelung des Benjamin'schen Zitats mit der Vorstellung eines kollektiven Urteilsspruches der durch eine solche Fotografie erteilt wird, wird zwar nicht behauptet, dass es sich auch dabei um einen Verweis auf Benjamin handelt, jedoch begibt sich diese Weiterführung gewissermaßen unter den, Schutz' des Zitates, profitiert von seiner Autorität, ohne dass ein solcher Zusammenhang im Ausgangstext auch nur angedeutet wäre.

Es entwickelt sich bei N. Richard ein Wechselspiel zwischen kreativer Lektüre Benjamin'scher Texte und ihrer zuweilen nicht unproblematischen Aufwertung zur poltisch-ästhetischen Autorität, die für eine Reihe von lateinamerikanischen Autoren charakteristisch ist. So wenn die auf die chilenische Militärdiktatur reagierende Literatur der 80er Jahre mit dem Urteil zusammengefasst wird:

Todas esas obras ilustraban la idea benjaminiana que , la continuidad de la historia es la de los opresores' mientras , la historia de los oprimidos es una discontinuidad': una sucesión inconclusa de fragmentos sueltos desamarrados por los cortes de sentido y que erran sin la garantía de una conexión segura ni de un final certero. (N. Richard, 26). 


\section{Erneuerung und Neuformulierung eines hermeneutischen Denkens; Rezeptionstheorie, Theorie der Übersetzung und Interkulturalität (M. Seligmann-Silva, Kampff-Lage)}

Neben einer postmodernen oder auch dekonstruktivistischen Lektüre Benjamins, die seine Texte und Reflexionen als, envoies als ,Sendungen in einem Prozess der Dissemination in einem durch Derrida legitimierten Verfahren aufnehmen, oder sie als Fragmente, wie in Collagen oder Montagen, in ihre eigenen Argumentationen einfügen, steht ein eher traditioneller Umgang mit seinen Texten, der um die Neuformulierung einer Hermeneutik, einer nicht europazentrieten, d.h. einer dezentrierten Hermeneutik, bemüht ist, und dafür ihn selbst als Legitimationsinstanz aufruft.

In einer philologisch anspruchsvollen Interpretation deckt Márcio SeligmannSilva in Ler o Livro de Mundo auf, in welchem Maße die Benjamin'sche Geschichtsund Sprachphilosophie dem romantischen Denken Friedrich Schlegels und Novalis' verpflichtet ist und dass vom Begriff der Kunstkritik her nicht nur der frühe Benjamin zu begreifen ist, sondern dass sich von diesem Begriff aus eine innere Kohärenz herstellen lässt, die sich durch alle Texte Benjamins zieht. Vom romantischen Begriff der Kunstkritik aus öffnet sich Benjamin dann auch, darauf weißt Seligmann-Silva hin, der Anschluss zur post-modernen Benjamin-Rezeption (Seligmann-Silva 76). Allerdings hält Seligmann-Silva an einem Benjamin fest, dessen Denken um Fragen der Hermeneutik und der Schriftauslegung kreisen, der einen Begriff der Wahrheit nicht aufgibt und dessen Begriffe der Allegorie und der Allegorese die immer im Zusammenhang des Herausstellens eines verborgenen Sinnes zu begreifen sind, seine Vereinnahmung durch einen modischen Konstruktivismus im Grunde unmöglich machen (cf. S. 213). Konstruktion im benjaminschen Sinne ist immer Interpretation einer uns nicht zugänglichen Wahrheit.

Überraschend ist für den europäischen Leser an dieser Arbeit, die sich ausführlich mit der europäischen Benjamin-Diskussion auseinandersetzt vielleicht nur, dass die lateinamerikanische Benjamin-Literatur fast ganz unberücksichtigt bleibt.

Seligmann-Silva ist damit keine Ausnahme in der lateinamerikanischen BenjaminRezeption. Ähnliches gilt, wie bereits gesagt, für die Untersuchung des chilenischen Philosophen Pablo Oyarzún, oder auch für den Argentinier Ricardo Ibarlucia. Auch dessen Untersuchung zu dem kleinen Essay Onirokitsch von W. Benjamin steht in einer ,klassischen' philosophisch-philologischen Tradition der Interpretation. Mit grosser Kenntnis der entsprechenden wissenschaftlichen Bibliographie werden die Bezugnahmen W. Benjamins auf die französischen Surrealisten freigelegt und aufgedeckt, inwieweit es sich bei diesem Text um eine Vorstudie zu dem berümten Surrealismus-Aufsatz von W. Benjamin handelt. Dennoch wäre interessant, der Frage nachzugehen, ob sich in diesen Untersuchungen nicht doch, unterschwellig' eine signifikative Verschiebung ausmachen lässt, die in ihnen selbst nicht thematisiert ist, die aber doch einem lateinamerikanischen Diskussionszusammenhang zuzuschreiben sind. Bei Ibarlucia z.B. fällt auf, dass hier eine Auseinandersetzung mit den grossen französischen Surrealisten Breton, Aragon, Tristan Tzara, wie auch mit den deutschen Romantikern nicht aufgrund eines primären Interesses für diese Autoren stattfindet, sondern vielmehr ist Benjamin der Anlass, um diese Autoren zu diskutieren. Von Benjamin aus wird also eine Perspektive auf die europäische Literatur- und Philosophiegeschichte eröffnet, von Benjamin aus wird ein europäischer Kontext entworfen und nicht wird, wie im Falle der europäische Kritik, Benjamin in einen bereits existierenden europäischen Kontext gestellt. Er wird zum Ausgangspunkt von 
dem aus die Auseinandersetzung mit europäischen Autoren und literarischen Traditionen stattfindet und diese werden in dem Maße, wie sie bei ihm thematisiert sind, in den Blick gerückt. Das ist besonders auffällig im Fall des Trauerspiel-Buches, dessen Lektüre einzig dem Namen Benjamins und nicht einem Interesse am deutschen Trauerspiel des Barock zuzuschreiben ist ${ }^{23}$.

Einen ganz anderen Vorschlag hermeutischer Rekonstruktion der Einheit des Benjamín'schen Denkens legt Susana Kampff Lages mit Walter Benjamin, Tradição e Melancolia vor, in dem sie Max Pensky's Melancholy Dialectics. Walter Benjamin and the Play of Mourning aufgreift und weiterentwickelt (cf. Kampff Lages, 101) und unter dem Begriff der Melancholie bei aller Vielseitigkeit seines Denkens seine innerere Einheit aufzudecken versucht. Allerdings nähert sie sich in ihrem argumentativen Vorgehen eher dem postmodernen Denken und legt dann auch in Benjamins ,Aufgabe des Übersetzers "uma espécie de arcano da teoria pós-moderna da tradução" (Kampff Lages, 233) frei. Das wird bereits in ihrer Grundidee deutlich, in ihrer Verkopplung von Melancholie und Übersetzung. Zwischen beiden Begriffen stellt sie eine Ähnlichkeit im Benjamin'schen Sinne her, eine Ähnlichkeit, die nicht auf der Ebene der sinnlichen Erscheinung zu identifizieren ist, sondern Bezug nimmt auf eine Parallelsierung des Verhältnisses des Melancholikers zur Welt und des Übersetzers zu seinem Ausgangstext: beide sind gekennzeichnet durch ein Oszillieren zwischen den Extremen der Verzweiflung über die absoluten Unerreichbarkeit und die narzistischen Überschätzung, die ,Vorlage' übertroffen zu haben (Kampff Lages, 232). Auf diese Weise gelingt es Kampff Lages eine Korrespondenz herzustellen zwischen literaturtheoretischen Aussagen zur Allegorie, Sprachtheorie und Benjamin'scher Lebenserefahrung und - im Grunde, etwas paradox für eine postmoderne Argumentation, so scheint mir -, damit eine innere Einheit von Leben und Denken bei Benjamin aufzudecken.

\section{Postmoderne und postkoloniale Kritik.}

Das Buch von Kampff Lages haben wir hier bereits als Übergang zwischen einer hermeneutischen und einer postmodernen Benjamin-Lektüre situiert. Denn geradezu als anderes Extrem zu einer Benjamin-Lektüre, die sich in eine 'universelle' BenjaminRezeption einschreibt, kann der Bezug auf seine Texte im Umkreis der lateinamerikanischen Postmoderne, der postkolonialen Kritik und der 'estudios culturales / cultural studies' angesehen werden. Während eine Untersuchung im Stile von der Seligmann-Silvas nicht die historischen und geographischen Koordinaten markiert, von denen aus sie geschrieben wurde (auch bei Kampff Lages ist dies nicht der Fall) und von denen aus sie argumentiert, legt die postkoloniale Kritik gerade darauf Wert, ihren Standort, Lateinamerika, von dem aus und für den sie schreibt ganau anzugeben. Ihr geht es vorzugsweise um eine Aktualisierung und um eine Adaptierung Benjamins für den lateinamerikanischen Kontext.

In diesem Zusammenhang sollen hier vor allem drei bereits oben in anderem Zusammenhang erwähnte AutorInnen nochmals aufgeführt werden: Beatriz Sarlo, Nelly Richard und Martín-Barbero. In den theoretischen Überlegungen, für die diese

\footnotetext{
${ }^{23}$ Aber auch hier bilden die lateinamerikanischen Intellektuellen keine Ausnahme. Die intensive theoretische Rezeption eines Bachtin, eines Lotmann oder der deutschen Slavistin Renate Lachmann findet auch in der Regel statt, ohne dass eine profundere Kenntnis der Texte anhand deren theoretische Aussagen gewonnen wurden, vorhanden ist.
} 
Intellektuellen hier als Repräsentanten zitiert sind, stehen die Fragen zu Urbanität, zu Erinnerung und Gedächtnis und $\mathrm{zu}$ Massenkultur und Kulturindustrie in dem umfassenderen Zusammenhang der Herausarbeitung einer dezentrierten Kulturtheorie, der Desterritorialsierung einer okzidentalen, oder genauer vom Norden dominierten Theorie und ihrer Reterritorialisierung in den Regionen, denen vorher nach dem Modell Zentrum - Peripherie ein blosser Satelitten-Status zukam. Benjaminsche Reflexionen von der "erlösten Menschheit (der) ihre Vergangenheit in jedem ihrer Momente zitierbar geworden (ist)" (Benjamin, Bd. 2, 694. These III) oder "Denn was er (der historische Materialist $\mathrm{HN}$ ) an Kulturgütern überblickt, das ist ihm samt und sonders von einer Abkunft, die er nicht ohne Grauen bedenken kann. Es dankt sein Dasein nicht nur der Mühe der großen Genien, die es geschaffen haben, sondern auch der namenlosen Fron ihrer Zeitgenossen.” (Benjamin, Bd. 2, 696. These VII) bekommen unter dieser Perspektive ihre ganz eigene Aktualität. Was bei Benjamin darauf angelegt ist, die europäische Kulturgeschichte neu zu denken, Denkbilder $\mathrm{zu}$ entwerfen, dialektische Bilder, die das 'Kontinuum der Geschichte aufsprengen' wird von der von Benjamin inspirierten postkolonialen Kritik gegen den 'Eurozentrimus' und seine Ideologie des Fortschritts ins Feld geführt.

Während 'traditionelle' Rezeption im Stile von P. Oyarzún, Ibarlucia oder Seligman-Silva Benjamin in den akademischen Diskurs einschreiben, wird hier ganz programmatisch eine anti-akademische Rezeption betrieben, ja - und das ist nicht nur polemisch formuliert - ein Benjamin-Recyling unternommen:

So lesen wir bei Nelly Richard:

Las obras chilenas entraron en connivencia con los textos de W. Benjamin saltándose muchas veces los relevos del saber universitario, entrelazando sus claroscuros sin pasar por la mediación académica de una cadena de pensamineto formalmente diseñada. Lo hicieron más bien inspiradas por ciertas alianzas de parentescos que se acordaban secretamente, sin órdenes de programas ni métodos. Una mezcla de azares y necesidades terminó haciendo productivas varias referencias benjaminionas, pasando por 'las combinaciones, las permutaciones, las utilizaciones' de conceptos cuya pertinencia y validez 'no son nunca interiores, sino que dependen de las conexiones con tal o cual exterior' [Zitat aus: Gille Deleuze und Félix Guattari: Rizoma (1976), Valencia: Pre-textos, p. 60] tal como lo señalan Deleuze-Guattari en su defensa de la experimentalidad del sentido." (Richard 1999 a, 15).

Auch der Blick auf die Massenkultur und die Kulturindustrie, auf die Fragmente und auf die 'Abfälle' der Kultur, die zum Programm von nirgends genau definierten 'cultural studies' geworden sind, kann sich vielfach auf Benjaminsche Textstellen berufen und Martín-Barbero konstatiert: 
obra de Benjamin en América latina adquirió estatus estratégico durante los años setenta, en Brasil incluso antes. Benjamin (1973) fue uno de los primeros en asumir - desde la diferencia radical entre narración y novela - la pluralidad y alteridad de las escrituras modernas. Frente a una concepción unificadora de lo cultural, Benjamin pensaba los márgenes de la modernidad y, lo que hoy es más interesante, reflexionaba desde ellos. (MartínBarbero / Herlinghaus, 47). ${ }^{24}$

Der siegreiche Einzug, den Benjamin in die 'estudios culturales' gehalten hat ohne dass er dafür selbst veranwortlich gemacht werden könnte - wird allerdings auch durchaus kritisch gesehen:

\begin{abstract}
En los alambiques de la Internacional académia, promotra de los últimos desarrollos industriales de los estudios culturales, se combina, con envidiable secillez, a Foucault con Benjamin, a Derrida con Deleuze y a Ramond Willimas von Bajtin. Todo suma. Sin embargo, la suma es el problema. Los usos de Benjamin como teórico de los estudios culturales y como teórico de un catecismo para aficionados a la ciudad moderna han llegado a su límite. (Sarlo, 86).
\end{abstract}

Und im gleichen Artikel: "Todo el mundo habla Benjamin, todo el mundo habla hoy el esperanto de los estudios culturales. " (Sarlo, 87).

Wie bereits zu Beginn herausgestellt wurde, können im Rahmen eines solchen Aufsatzes nur einige Fakten zusammengetragen werden, die auf der einen Seite erlauben einen ersten Eindruck vom Umfang und von der Bedeutung der BenjaminRezeption in Lateinamerika zu geben und die auf der anderen Seite versuchen einige Vermutungen $\mathrm{zu}$ belegen, welches die Gründe und die Voraussetzungen dieser Rezeption sind.

Abschließend sollen nun noch einige weitere Fragestellungen im weiteren Umkreis der Benjamin-Rezeption wenigstens kurz angeschnitten werden.

\title{
Die Benjamin-Übersetzungen
}

Wie bereits erwähnt erschienen die ersten Benjamin-Übersetzungen ins Spanische und Portugiesische Ende der 60er Jahren im Umfeld der nicht-dogmatischen

\footnotetext{
${ }^{24}$ Dabei handelt es sich durchaus um ein 'lateinamerikanisches Phänomen'. Wenn wir den Band Jenseits des Eurozentrismus. Postkoloniale Perspektiven in den Geschichts- und Kulturwissenschaften, herausgegeben von Sebastian Conrad und Shalini Randeria, Frankfurt / New York, 2002: Campus, durchsehen, stellen wir fest, dass in den elf daran versammelten Aufsätzen von Wissenschaftlern aus Deutschland und US-Amerika mit jeweils sehr ausführlichen Bibliographien, Benjamin nirgends zitiert und nur bei Timothy Mitchell in „Die Welt als Ausstellung“(148-176), der Aufsatz „Paris, die Hauptstadt des XIX Jahrhunderts“ in der Bibliographie erwähnt wird.
} 
Theoriediskussion einer neu sich formierenden Linken ${ }^{25}$. Eine Untersuchung zu diesen Übersetzungen, zu den unterschiedlichen Varianten gleicher Benjamin-Texte, die es erlauben würde die Schwierigkeiten zu analysieren mit denen Benjamin-Übersetzer konfrontiert sind, steht noch aus. Hier können zu dieser Frage nur einige allgemeine Anmerkungen formuliert werden. Zweifellos bereitet das 'Über-setzen' von BenjaminTexten Schwierigkeiten, die über die gewöhnlichen Probleme, durch die jeder Übersetzer herausgefordert wird hinaus gehen. Neben gewissen Unzulänglichkeiten, Ungenauigkeiten oder auch Missverständnissen, die sich in den BenjaminÜbersetzungen finden ${ }^{26}$, ergibt sich die Hauptschwierigkeit für den Übersetzer/die Übersetzerin aus der Benjamin'schen Sprachphilosophie selbst, nach der die Sprache nicht ein Werkzeug ist, um einen Gedanken oder eine Theorie darzustellen, sondern das Denken durch die Sprache selbst seinen Ausdruck findet. In diesem Sinne verlangt die Übertragung in eine andere Sprache ein 'Über-setzen' des Denkens selbst, insofern nach Benjamin die Sprache dem Denken nicht äusserlich ist, sondern beide sich gegenseitig bedingen da die Sprache immer um eine 'mimikry' des Denkens bemüht ist. Das ist wohl einer der Hauptgründe für eine gewisse Hermetik der Benjamin'schen Texte und für die Hindernisse, die sie der Übersetzung in andere Sprachen entgegenstellen. Sie müssen gewisseremassen in der anderen Sprache neu gedacht werden. Die wörtlichen Übersetzungen sind deshalb vielfach die am wenigsten genauen. Benjamin hat das in seinen eigenen Übersetzungsvorschlägen, unter anderem der Gedichte Baudelaires gezeigt, in denen er sich oftmals für Lösungen entscheidet, die ausgefallen und dem deutschen Sprachgebrauch fremd erscheinen, um den Ideen der Baudelaire'schen Vorlage gerecht zu werden ${ }^{27}$.

Wie Jeanne Marie Gagnebin uns in ihrer Einleitung zu dem Buch von S. Kampff Lages, Walter Benjamin, Tradução e Melancolia erinnert, ist die Aufgabe einer Übersetzungen der Benjamin'schen Texte immer 'Aufgabe' in der dreifachen Bedeutung des deutschen Wortes:

1. 'Aufgabe' als ethische und politische Aufgabe der Übertragung

2. 'Aufgabe' als Verzicht, als Entsagung und als Einsicht, dass die Sprache nicht (mehr) alles das zu sagen vermag, was wir ihr zumuten und

\footnotetext{
${ }^{25}$ Wenngleich der Name Walter Benjamin offensichtlich schon vorher in literarischen Kreisen zirkulierte. Kampff Lage zitiert ein Interview (nach: F. Camacho: „Entrevista com João Guimarães Rosa“, p. 52) mit João Guimarães Rosa, in dem dieser kurz vor seinem Tod (1967) Walter Benjamin (neben Júlio Dantas, Fernando Camacho, Goethe, Rubem Braga, Magalhaes Júnior, Machado de Assis, Eça de Queirós) als einen der Autoren angibt, der in seine Literatur Eingang gefunden hat: ,... entra tanta coisa, ficando tudo misturado. O que entra eu junto com (...) Júlio Dantas, Fernando Camacho, Walter Benjamin, Goethe, Rubem Braga, Magalhães Júnior, Machado de Assis, Eça de Queirós.” (Kampff Lages, 126)

${ }^{26}$ So zum Beispiel das Ende der Übersetzung des bekannten Aufsatzes über den Sürrealismus, in der die spanische Version das Benjamin'sche Bild auch nicht annähernd trifft:

„Sie geben, Mann für Mann, ihr Mienenspiel in Tausch gegen das Zifferblatt eines Weckers, der jede Minute sechzig Sekunden lang anschlägt.“(Benjamin, Bd. 4, 310)

${ }^{27}$ In "Die Aufgabe des Übersetzers" bezieht sich W. Benjamin auf die Übersetzung 'literarischer Werke'. In dem Maße allerdings, in dem für Benjamin ,Literaturkritik' im Sinne der romantischen 'Kunstkritik' teil des Kunstwerks selbst wird, gilt für ihn ein Gleiches für die Übersetzung. Wobei diese These dann konsequenterweise auf die Übersetzung von Benjamins eigenen Schriften auch Anwendung finden muß: "Übersetzungen, die mehr als Vermittlungen sind, entstehen, wenn im Fortleben ein Werk das Zeitalter seines Ruhms erreicht hat. Sie dienen darher nicht sowohl diesem, wie schlechte Übersetzer es für ihre Arbeit zu beanspruchen pflegen, als dass sie ihm ihr Dasein verdanken. In ihnen erreicht das Leben des Originals seine stets erneute späteste und umfassende Entfaltung. » (Walter Benjamin : Bd. 10, p. xxx ) Hier wird also, in einer provokativen Wende, die Übersetzung höher als das Original veranschlagt, weil es in dieser eine ,erneute späteste und umfassende Entfaltung“ findet.
} 
3. 'Aufgabe' im Sinne der Subjektivierung des Verbes 'aufgeben', im Sinne der idiomatischen Wendung 'einen Brief aufgeben', d.h. 'Auf-gabe' im Sinne der Derrida'schen 'envoie'. (cf. Kampff Lages, 17)

Im ersten Sinnhorizont erkennten wir eindeutig eine hermeneutische Position wieder, der zweite markiert gewissermassen den Übergang von einer hermeneutischen zu einer dekonstruktivistischen und postmodernen Position und der dritte ist eindeutig einer solchen dekonstruktivistischen und postmodernen Einsicht zuzuordnen.

\section{W. Benjamin in Forschung und Lehre}

Leider ist es in diesem Zusammenhang auch nicht möglich auch nur einen ungefähren Überblick über den heutigen Stand der Benjamin-Forschung in Lateinamerika zu geben. Es werden sich mit Sicherheit wenige Fakultäten im Bereich der Geisteswissenschaften an den lateinamerikanischen Universitäten finden lassen, an denen nicht in den letzten Jahren 'Memorias', Magisterarbeiten oder Doktorabeiten zu den Schriften Walter Benjamins vorgelegt wurden, oder zumindest Arbeiten, die mit einer umfangreichen Benjamin-Rezeption verbunden waren. Aufgrund der Vielseitigkeit seines Denkens sind diese Arbeiten nicht nur auf die Abteilungen für Literaturwissenschaft und Philosophie beschränkt, sondern finden sich auch bei den Übersetzungs-, den Kommunikations-, den Kulturwissenschaften oder bei Urbanisten.

Aus Anlass des hundertsten Geburtstages hatte das Goethe-Institut Buenos Aires $1992 \mathrm{zu}$ einem grossen Benjamin-Symposium eingeladen, in dem BenjaminSpezialisten aus Europa und Lateinamerika zu Wort kamen. Dass nur wenige der dort vertretenen Wissenschaftler hier Erwähnung gefunden haben ist ein weiterer Beweis für die Fülle und den Umfang der Benjamin-Rezeption. Auf die drei Beiträge, allerdings, die explizit die Benjamin-Rezeption in Lateinamerika zum Thema hatten, soll hier abschliessend noch eingegangen werden.

Graciela Wamba Gaviña legte einen historischen Überblick über die BenjaminRezeption in Argentinien vor und fasst seine Bedeutung für die intellektuelle Diskussion des Landes wir folgt zusammen:

En buena medida, la crítica sobre Benjamin en Argentina tiene el énfasis de una celebración cuyos objetos son las afinidades secretas, la pasión del coleccionista, la deriva del flâneur; el detalle aparentemente insignificante, el arte de la cita, el antiacademicismo, etc. Si bien no faltan ejemplos en los que el pensamiento de WB se toma como un material para ser repensado y desarrollado, también puede señalarse una amplia recepción que se abstiene de tomar su pensamiento como un contenido teórico; esta recepción se realiza de dos maneras o bien tomando su pensamiento como un mero atributo de WB o bien consinderándolo no como un producto sino como un método, como una forma de pensar la asistematicidad; el cruce de saberes distintos; la conjunción de campos teóricos heterogéneos; la inseparabilidad del pensamieneto y la dimensión poética de la escritura; la estrategia de elaborar teorías con conceptos inaprovechables para el enemigo; un pensamiento inicial que efectúa un trabajo de reconstrucción a partir de pequeñas 
huellas; la investigación concebida como un proyecto abierto; etc. Estos aspectos formales, que al mismo tiempoque explican el carácter inclasificable y fragmentario de WB constituyen, ellos mismos, lo más definido y constante de su pensamieneto, representan sin duda, para los lectores argentinos de WB, su herencia más fecunda. (Wamba Gaviña, Graciela, pp 213-214).

Das Panorama, das Leonidas Morales im Bezug auf die chilenische BenjaminRezeption der 70er und 80er Jahre zeichnet ist vollständig verschieden von dem Wamba Gaviñas für Argentinien. In den Jahren der Erneuerung der chilenischen Literaturkritik, 1960 - 1973 gilt das Interesse entweder dem französischen Strukturalismus oder einem Marxismus, der mit den Namen Lukács, Sartre und Goldmann verbunden ist. Während der Zeit der Militärdiktarur, bis Ende der 80er Jahre findet sich in den beiden bedeutenden Literaturzeitschriften der Universitäten Universidad de Chile und Universidad de Concepción kein einziger Verweis auf Benjamin. (cf. Morales, pp. 218219). In alternativen intellektuellen Kreisen bekommt der Name Benjamin mit Beginn der 80er Jahre einen gewissen Klang. Martín Cerda in La palabra quebrada ${ }^{28}$ ist nach Leonidas Morales wohl der erste chilenische Kritiker der über Benjamin schreibt. (Morales, 219) Erst mit dem Ende der Militärdiktatur, ab 1989, zeichnet sich eine Veränderung in den Rezeptionsbedingungen $\mathrm{ab}$ und $\mathrm{W}$. Benjamin findet wirklich Eingang in die chilenische Literaturkritik. Der endgültige Eintritt Chiles in die Modernität im Laufe der 90er Jahre machen nun W. Benjamin, so Morales, zu einem unverzichtbaren Theoretiker:

\footnotetext{
La crítica de cultura chilena y latinoamericana no puede prescindir, si quiere ser no sólo comprensiva sino además liberadora, de los lúcides análisis de Benjamin. Pero tampoco la crítica literaria. (...) No abordar los textos de frente, sino de costado. No con la lógica del centro, sino con la de la perifería, de los bordes. (Morales, 221).
}

Nochmals ein anderes Bild entwirft Günter Karl Pressler in seiner Studie über die brasilianische Benjamin-Rezeption. Hier ist seit den 60er Jahren eine stetige Kontinuität zu verzeichnen, die dazu geführt hat, dass der Philosoph heute ein mit Selbstverständlichkeit zitierter Name in den Feuilletons der grossen Brasilianischen Tageblätter ist. (Pressler, 223).

Pressler macht vier Phasen der Benjamin-Rezeption in Brasilien aus:

1. 1967-1969: Die Entdeckung des marxistischen W. Benjamin

2. 1975-1983: Veröffentlichungen der ersten ausgewählten in Brasilien übersetzten Texte. Benjamin als Theoretiker der Moderne.

3. 1984-1989: Veröffentlichung der Obras Escolhidas in drei Bänden und vor allem die Rezeption der Thesen über den Begriff der Geschichte.

\footnotetext{
${ }^{28}$ Cerda, Martín (1982): La palabra quebrada. Valparaíso: Ediciones Universitarias.
} 
4. 1990-1992: Ausweitung der universitären Forschung und Lehre über W. Benjamin über Rio de Janeiro und Sao Paulo hinaus. Symposium zu seinem 50. Todestag in Sao Paulo. (Pressler 226-227)

Ein weiteres offensichtliches Desiderat wäre es, jenseits der unmittelbaren Rezeption, der Adaptierung und des 'Recyclings' des Benjamin'schen Denkens, seine Wirkungen, Konsequenzen und kreative Umsetzungen aufzudecken und auf diese Weise in einer 'dichten Beschreibung' einige Maschen aus dem engen Netz intellektueller Beziehungen zwischen Lateinamerika und Europa freizulegen.

Bei den vorgestellten Texten handelte es sich immer um Beispiele, in denen Benjamin selbst thematisiert wurde. Die weiterreichende Fragestellung, in welchem Masse die gesamte lateinamerikanische Literatur- und Kulturkritik Impulse aus dieser Diskussion empfangen hat, in welchem Masse auch Kulturkritiker von der Bedeutung eines Carlos Monsivais ${ }^{29}$ oder eines Nestor García Canclini ${ }^{30}$, die sich meines Wissens nach nicht ausführlich zu W. Benjamin geäussert haben, implizit im Dialog mit W. Benjamin stehen, muss hier unbeantwortet bleiben. Ebenso wie hier unbeantwortet bleiben muß, was diese intensive Benjamin-Diskussion auf dem amerikanischen Kontinent für Europa, besonders aber für Deutschland zu bedeuten hat. Nicht nur in der Form einer Rückkoppelung und der Aufforderung, auch die Interpreationsvorschlägen dieser Region in der deutschen Benjaminforschung $\mathrm{zu}$ berücksichtigen, sondern vielmehr als Möglichkeit, anhand dieser Lektüren, Interpretationen und Transformationen eine Vorstellung und einen Begriff davon zu gewinnen, welche kulturellen, philosophischen und theoretischen Fragen in Lateinamerika verhandelt werden.

\footnotetext{
${ }^{29}$ So z. B. Bei Carlos Monsivais in "Profetas de un Nuevo Mundo. Vida urbana, modernidad y alteridad en América Latina (1880-1920)". In: Monsivais, Carlos (2000): Aires de familia. Cultura y sociedad en América Latina. Barcelona: Anagrama. pp. 181-210. Dieser Artikel, der an keiner Stelle W. Benjamín zitiert, scheint dennoch vielfach - vielleicht nur vermittelt über die von ihm stimulierte Kulturdiskussion der letzten Jahrzehnte - den Benjamin von Paris, die Hauptstadt des XIX Jahrhunderts und einige Motive bei Baudelaire auf Lateinamerika zu adaptieren.

${ }^{30}$ In Nestor García Canclinis berühmten Culturas hibridas (1990) México D.F.; Grijalbo, wird an einer Stelle wiederholt „Das Kunstwerk im Zeitalter seiner technischen Reproduzierbarkeit“ (185) und dann noch „Beim Auspacken meiner Bibliothek“ (282) zitiert. Ob und in welchem Masse Benjamin darüber hinaus implizit präsent ist kann hier nicht beantwortet werden.
} 


\section{Referências Bibliográficas}

1. Acha, Juan Omar: ¿Olividar a Benjamín? Historicidad e interpretación. Punto de Vista. Buenos Aires Núm.

2. Ballent, Anah, Adrián Gorelik, Graciela Silvestri: Las metrópolis de Benjamín. In: Punto de Vista núms.: 45, 53, 55. Buenos Aires; 1993, 1995, 1996.

3. Benjamín, Walter (1980): Über den Begriff der Geschichte. In: Benjamin, Walter (1980): Gesammelte Schriften, Werkausgabe, Bd. 2, Frankfurt: Suhrkamp, pp. 691 704.

4.-------------(1980): Der Sürrealismus. In: Benjamin, Walter (1980):

Gesammelte Schriften, Werkausgabe, Bd. 4, Frankfurt: Suhrkamp, pp $295-310$.

5.------------(1980): Kleine Geschichte der Photographie. In: Benjamin, Walter (1980): Gesammelte Schriften, Werkausgabe, Bd. 4, Frankfurt: Suhrkamp, pp 368 385.

6.----------(1980): Die Aufgabe des Übersetzers. In: In: Benjamin, Walter (1980): Gesammelte Schriften, Werkausgabe, Bd. 10, Frankfurt: Suhrkamp.

7. Bolle, Willi $(1994$, a) Fisiognomia da metrópole moderna : representacão da história em Walter Benjamin. São Paulo : Edusp Ed. da Univ.

8.----------- $(1994$, b): Physiognomik der modernen Metropole: Geschichtsdarstellung bei Walter Benjamin. Koeln, Weimar, Wien: Boehlau.

9. Casullo, Nicolas (1993). A modo de prólogo. Actualidad de Benjamin en América Latina. En: Fehrmann, Silvia y Gabriela Massuh (1993): Sobre Walter Benjamín. Vuanguardias, historia, estética y literatura. Una visión latinoamericana. Buenos Aires. Alianza Editorial /Goethe-Institut Buenos Aires. pp. 11-19

10.---------------. Walter Benjamín y la modernidad. Revista de Crítica cultural, No. 4, 1991.

11. Collingwood-Selby, Elizabeth (1997). Walter Benjamin. La lengua del exilio. Santiago de Chile. Libros ARCIS-LOM.

12. Gagnebin, Jeanne Marie (1994). Histoire et Narration chez Walter Benjamin, Paris: Harmattan.

13. (1999; 2nda edición revisada). História e narração em Walter Benjamin. São Paulo. Editora Perspectiva.

14. Garber, Klaus und Rehm, Ludger (Hgg) (1999). Global Benjamin: Internationaler Waler-Benjamin-Kongreß 1992. München: Fink 
15. García de Leon, Antonio (1997). El pasado-presente: a propósito del tiemp y el lenguaje de Walter Benjamin". En: Aproximaciones a la modernidad. Paris Berlin. Siglos XIX y XX. Ed. Patricia Nettel y Sergio Arroyo. Universidad Autónoma Metropolitana-Xochimilco. México. Pp 69-90.

16. Goebel, Rolf J. (1999): „Postkoloniale Kritik und kulturelle Authentizität. Zur Rezeption Walter Benjamins in der amerikanischen Kulturtheorie.“ Weimarer Beiträge 45, 4. pp. 532-546.

17. Ibarlucía, Ricardo (1997a). Walter Benjamin y la génesis de Passagen-Werk. En : Revista Latinoamericana de Filosofía, Vol XXIII, N.2, pp. 319-343.

18.----------------(1997b). Walter Benjamin: iluminacão histórica e interpretacão dos sonhos na gênesis de "Passagen-Werk" In: Revista USP. Nr. 33: Dossiê Aids São Paulo 1997 Nr. 22, S. 172-186.

19. (1998). Onirolkitsch. Walter Benjamin y el surrealismo. Buenos Aires. Manantial.

20. Kampff Lage, Susana (2002). Walter Benjamin: Tradução e Melancolia. São Paulo. Editora da Universidade de São Paulo.

21. Konder, Leandro (1967). Os Marxistas e a Arte. Rio de Janeiro: Civilização Brasileira.

22.(1988). Walter Benjamin: o marxismo da melanconia. Rio de Janeiro: Campus.

23. Kothe, Flavio René (1976). Para ler Benjamin. Rio de Janeiro: Alves Ed.

24. Lowy, Michel (1990). Romantismo e messianismo: ensayos sobre Lukács e Walter Benjamín. Sao Paulo. Perspectiva.

25. Martín-Barbero, Jesús (1987). De los medios a las mediaciones. Barcelona: G. Gili.

26. (2000). Mis encuentros con Walter Benjamin. En : Jesús

Martín-Barbero / Hermann Herlinghaus : Contemporaneidad latinoamericana y análisis cultural. Conversaciones al encuentro de Walter Benjamin. Frankfurt. Pp. 11-22.

27.-----------------(2000). La crisis del hermeneuta o perspectivas transdisciplinares de la modernidad. En: Jesús Martín-Barbero / Hermann Herlinghaus : Contemporaneidad latinoamericana y análisis cultural. Conversaciones al encuentro de Walter Benjamin. Frankfurt. pp. 41-62.

28. (2000). Modernidades y destiempos latinoamericanos. En : Jesús Martín-Barbero / Hermann Herlinghaus : Contemporaneidad latinoamericana y análisis cultural. Conversaciones al encuentro de Walter Benjamin. Frankfurt. pp. 84104. 
29. Merquior, José Guilherme (1969). Arte e Sociedade em Marcuse, Adorno e Benjamin. ORT VERLAG.

30. Monsivais, Carlos (2000). Profetas de un Nuevo Mundo. Vida urbana, modernidad y alteridad en América Latina (1880-1920). En: Monsivais, Carlos: Aires de familia. Barcelona: Anagrama. pp. $181-210$.

31. Morales T., Leónidas (1993). Walter Benjamin y la crítica literaria chilena. En: Fehrmann, Silvia y Gabriela Massuh (1993). Sobre Walter Benjamín. Vuanguardias, historia, estética y literatura. Una visión latinoamericana. Buenos Aires. Alianza Editorial /Goethe-Institut Buenos Aires. pp 215-222. Wiederabgedruckt in: Morales T., Leonidas (2001): La escritura de al lado. Santiago. Cuatro propio. pp. Xxx - xxx.

32. Muricy, Katia (1999). Alegorias da diáletica: imagem e pensamento em Walter Benjamin. Rio de Janeiro : Relume Dumará, 1999.

33. Ortiz, Renato (1988): A moderna tradiçao brasileira. Cultura Brasileira e Indústria Cultural. Sao Paulo: Editora brasiliense.

34.-------(2000 a). Walter Benjamín e Paris. Individualidade e trabalho intelectual. En: Tempos Social. Revista sociológica. Sao Paulo. USP 12 (1) pp. 11-28.

35.-----------(2000 b). Modernidad y Espacio. Benjamin en Paris. Bogotá: Grupo Editorial Norma. (Der Teil 'Benjamin en Paris' ist eine Übersetzung von Renato Ortiz 2000 a).

36. Otte, Georg (1996). Rememoracão e citacão em Walter Benjamin. In: Revista de estudos de literatura Belo Horizonte 1996; Vol. 4, S. 211-223.

37. Oyarzún Robles, Pablo (s.a.). Cuatro señas sobre experiencia, historia y facticidad. A manera de introducción (a la traducción de El concepto de la historia por P. Oyarzún Robles HN). En: Walter Benjamín (s.a.): La dialéctica en suspenso. Fragmentos sobre la historia. Santiago de Chile. Arcis.

38. (1999 a). Sobre el concepto benjaminiano de traducción (1990). En: Oyarzón R. Pablo: De lenguaje, historia y poder. Nuevos ensayos sobre filosofía contgemporánea. Santiago de Chile. Colección Teoría. Departamento de Teoría de las Artes. Facultad de Artes. Universidad de Chile. pp. 161 - 205.

39 (1999 b). Cuatro señas sobre experiencia, historia y facticidad en el pensamiento de Walter Benjamin. (1993, 1995). En: Oyarzón R. Pablo: De lenguaje, historia y poder. Nuevos ensayos sobre filosofía contgemporánea. Santiago de Chile. Colección Teoría. Departamento de Teoría de las Artes. Facultad de Artes. Universidad de Chile. pp 207 - 246.

40. Pressler, Günter Karl (1993). El perfil de los hechos. Sobre la recepción de Walter Benjamín en el Brasil. En: Fehrmann, Silvia y Gabriela Massuh (1993): Sobre Walter Benjamín. Vuanguardias, historia, estética y literatura. Una visión latinoamericana. Buenos Aires. Alianza Editorial /Goethe-Institut Buenos Aires. pp. 223-233. 
41. Richard, Nelly (1994 a). Roturas, memoria y discontinuidades (homenaje a Benjamin). En: La insubordinación de los signos. Cambio político, transformaciones culturales y poéticas de la crisis. pp. 13-36.

42. (1994 b). Destrucción, reconstrucción y desconstrucción. En: La insubordinación de los signos. Cambio político, transformaciones culturales y poéticas de la crisis. pp xxx.

43. Rouanet, Sérgio Paulo (1984). Introdução a Walter Benjamin. In: Chauí, Marilena de Souza (org.) Origem do drama barroco alemão. São Paulo. Brasiliense. pp. Xx - xx.

44. (1993). A Razão nomade. Walter Benjamin e outros viajantes. Rio de Janeiro.

45. (1997). Die Rezeption der deutschen Philosophie in Brasilien : der Fall Walter Benjamin. In: Andreas Boeckh; Rafael Sevilla (Hrsg.): Bestandsaufnahme und Perspektiven der deutsch-brasilianischen Beziehungen : 1. Fachtagung der ADLAF-Arbeitsgruppe Brasilien (Konstanz, 21.-23. Juni 1996) Frankfurt am Main. pp. 31-61.

46. Sarlo, Beatriz (1995). « Olvidar a Benjamin » Reproducido en: Sarlo, Beatriz (2000): Siete ensayos sobre Walter Benjamin. Buenos Aires. Fondo de Cultura Econômica; 1995.

47. (2000). Siete ensayos sobre Walter Benjamin. Buenos Aires. Fondo de Cultura Económica.

48. Seligmann-Silva, Márcio (1999): Ler o livro do mundo. Waler Benjamin: Romanismo e Crítica Poética. São Paulo. Editora Iluminuras Ltda.

49. Sobrevilla, David (1986): Repensando la tradición occidental. Lima

50. Walter, Monika (2000): Regreso de Walter Benjamin a los tiempos del Sur... In: En : Jesús Martín-Barbero / Hermann Herlinghaus : Contemporaneidad latinoamericana y análisis cultural. Conversaciones al encuentro de Walter Benjamin. Frankfurt. pp 175-181.

51. Wamba Gaviña, Graciela (1993): La recepción de Walter Benjamin en la Argentina. En: Fehrmann, Silvia y Gabriela Massuh (1993): Sobre Walter Benjamín. Vuanguardias, historia, estética y literatura. Una visión latinoamericana. Buenos Aires. Alianza Editorial /Goethe-Institut Buenos Aires. pp. 201 - 214.

52. Wesseling, Klaus-Gunther (2003): Walter Benjamín. Eine Bibliographie. Norhausen: Traugott Bautz. 